\title{
Surprising separation selectivity of ethylene from ethane over pure siliceous zeolites with framework flexibility
}

\author{
Xiangju Meng and Feng-Shou Xiao*
}

Recovery and purify of light olefins from the off-gas of the catalytic crackers have been focused for more than 50 years, driven largely by the escalating demand for feedstock for production of polyethylene and polypropylene, which requires the separation of $\mathrm{C}_{2} \mathrm{H}_{4} / \mathrm{C}_{2} \mathrm{H}_{6}$ and $\mathrm{C}_{3} \mathrm{H}_{6} / \mathrm{C}_{3} \mathrm{H}_{8}$ mixtures in which the olefins and paraffins are present at roughly equal concentrations $[1,2]$. Distillation operated at low temperatures (cryogenic distillation) or high pressure is one of the most useful methods for the separation and purify of these light olefins. However, these processes are energetically inefficient and therefore costly since the differences in volatility between the olefins and corresponding paraffins are so small, which require the separation column operated at high reflux, with more than 100 plates [2]. As a consequence, more than $0.3 \%$ of the global energy was consumed in the separation of ethylene and propylene from the corresponding paraffins. For the sake of energy-saving and cost-reducing, developing the efficient adsorption systems has been regarded as one of alternative technologies. However, no economically viable adsorptive separation process has yet emerged up to now, due to the relatively strong interaction between the olefins and selective adsorbents, which results in the complex regeneration process. As a result, new kinetically selective adsorbents for light olefins separation have been paid much attention in recent years.

Porous materials are promising candidates for efficient separation of gas mixtures by distinguishing the differences in molecular sizes, shapes, and polarities. Recently, custom-designed porous materials, in termed of metalorganic frameworks (MOFs) and/or porous coordination polymers (PCPs) with open lattices formed from inorganic centers (nodes) and organic linking groups have been paid much attention due to their inherent diversity, which afford precise control over pore chemistry and pore size and consequently excellent performance in gas separation or capture such as ethylene [3] and 1,3-butadiene [4]. However, the low thermal stability still limits their wide applications in the separation of light olefins from corresponding paraffins since thermal treatments are always required for regeneration of the adsorbents once the pores are blocked.

Zeolites, especially pure siliceous zeolites with suitable microporous structure would be ideal adsorbents for the separation of light olefins with paraffins because of their high thermal stability for regeneration and neutral framework for olefins non-oligomerization. Previous literatures have confirmed that pure siliceous 8-membered ring (MR) zeolites, e.g., CHA or deca-dodecasil 3R (DD3R) have shown superior performance on the separation of propylene and propane [1]. But the suitable zeolites for separation of ethylene and ethane are still a great challenge.

Excitingly, a joint team lead by Corma, Corcoran, Rey, and Ravikovitch [5] recently reported new pure siliceous zeolite (ITQ-55), which could kinetically separate ethylene from ethane with an unprecedented selectivity of $\sim 100$, owing to its distinctive pore topology with large heart-shaped cages and framework flexibility. $\mathrm{N}^{2}, \mathrm{~N}^{2}, \mathrm{~N}^{2}$, $\mathrm{N}^{5}, \mathrm{~N}^{5}, \mathrm{~N}^{5}, 3 \mathrm{a} 6$-octamethyloctahydropentalene-2,5-diammonium cation was employed as the organic structuredirecting agent (OSDA) in alkaline conditions $\left(\mathrm{OH}^{-}\right.$ media) or $\mathrm{F}^{-}$anions as mobilizer agents of the silica $\left(\mathrm{F}^{-}\right.$ media) to prepare the pure silica zeolites ITQ-55 constructed by twined heart-shaped cages that are interconnected by sharing a common 8-ring (8R) (ring aperture: 5.3 by $3.4 \AA$ ) as shown in Fig. 1a and b. These cavities are accessible through two parallel systems of zigzag 8R channels (ring aperture: 5.9 by $2.1 \AA$ ), sug- 


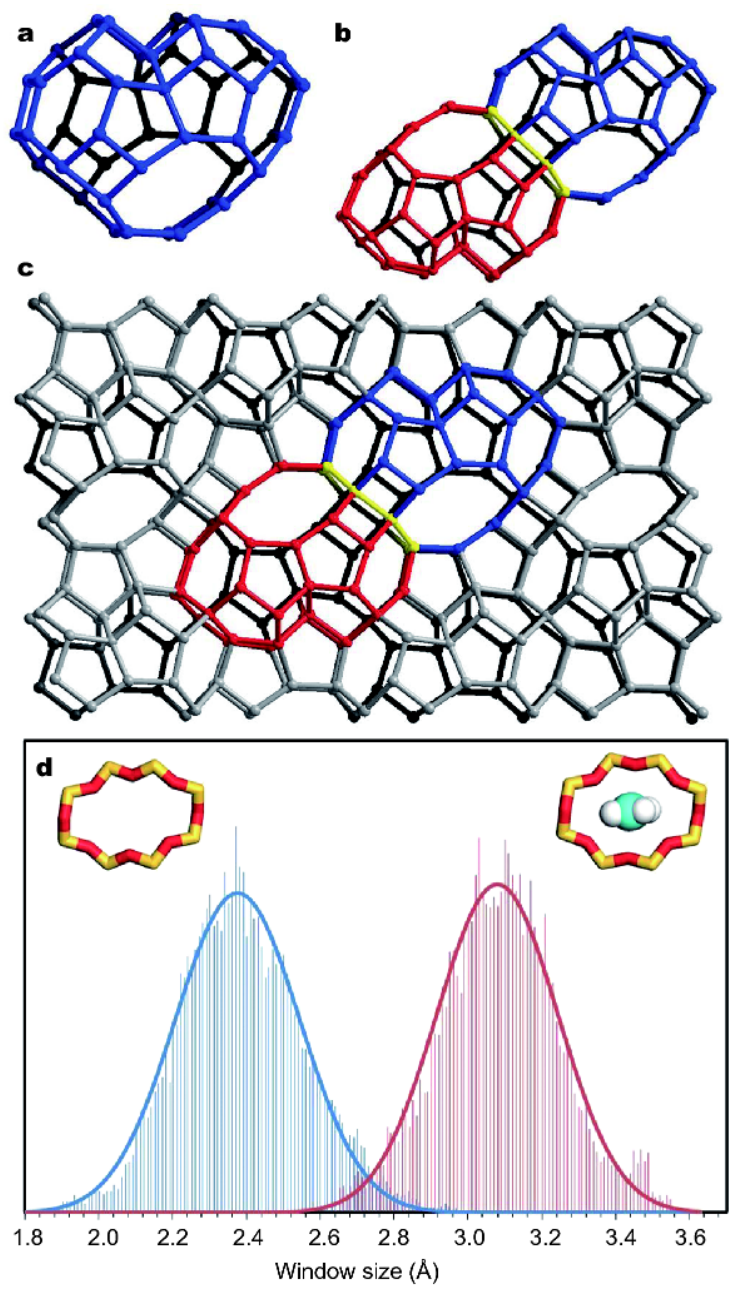

Figure 1 Description of the zeolitic structure of ITQ-55. (a) A 48T heart-shaped cage. (b) Dimeric $48 \mathrm{~T}$ cages. The yellow ring indicates the interconnecting 8-ring (8R). (c) Projection along the $b$ axis of the ITQ55 structure (oxygen atoms omitted for clarity; Tatoms in gray; two of the heart-shaped cavities highlighted in blue and red, respectively, for clarity). (d) ITQ-55 window sizes. Distributions of the minimal $8 \mathrm{R}$ window size for the empty structure of ITQ-55 (left) and the ITQ-55 structure with ethylene molecules constrained to the center of the $8 \mathrm{R}$ window (right), as calculated from the DFT molecular dynamics simulations at $300 \mathrm{~K}$. These pictures are from reference [5]. Copyright 2017, the American Association for the Advancement of Science.

gesting that ITQ-55 owns a tortuous monodirectional small-pore system with relatively large cavities (Fig. 1c). Furthermore, the size of the smallest pore window was calculated to be 2.33 by $5.71 \AA$, which is relatively comparable to the experimental size ( 2.07 by $5.86 \AA$ ). It is well known that the minimum diameter of the 8-ring, rather than the average diameter, is the critical factor for the gas separation. Such small pores would not adsorb either of these hydrocarbons, if the structure was rigid. The most exciting feature of ITQ-55 is the flexibility of the structure and $a b$ initio molecular dynamics (AIMD) simulations reveal that the mean window size is $2.38 \AA$ for the empty structure in agreement with the above results, and the mean window size increases to $3.08 \AA$, when ethylene molecules were constrained to the center of the smallest 8R window as shown in Fig. 1d, which indicates that $\mathrm{C}_{2} \mathrm{H}_{4}$ molecule "braces the window open" during molecular diffusion. As expected, the rate of ethylene adsorption on ITQ-55 is much higher than the corresponding adsorption rate of ethane and smaller ITQ-55 crystals achieve faster transport rates with even greater selectivity of $\sim 300$ in the separation of $\mathrm{C}_{2} \mathrm{H}_{4}$ and $\mathrm{C}_{2} \mathrm{H}_{6}$.

What is clear from this paper is that there is a great move towards the reduction of the energy consuming in the separation of $\mathrm{C}_{2} \mathrm{H}_{4}$ and $\mathrm{C}_{2} \mathrm{H}_{6}$. But can such processes be scaled up so that they have commercial significance? Any new process and zeolite production has challenges associated with scale-up, e.g., the cost and the calcination process of the SDAs [6], and one of the solution for these challenges is to synthesize the ITQ-55 in the absence of organic templates [7]. Of course, what has not been yet demonstrated is that the properties of thin ITQ-55 membranes are at least as good as those of the crystals powders. The fabrication of zeolite thin membranes is always a huge challenge for the zeolite synthesis. The fact that new zeolite of ITQ-55 throw up so many questions emphasizes that ITQ-55 opens a new door for fundamental studies of zeolites with framework flexibility for gas separation.

Received 23 December 2017; accepted 25 December 2017; published online 16 January 2018

1 Ruthven DM, Reyes SC. Adsorptive separation of light olefins from paraffins. Microporous Mesoporous Mater, 2007, 104: 59-66

2 Eldridge RB. Olefin/paraffin separation technology: a review. Ind Eng Chem Res, 1993, 32: 2208-2212

3 Cui X, Chen K, Xing H, et al. Pore chemistry and size control in hybrid porous materials for acetylene capture from ethylene. Science, 2016, 353: 141-144

4 Liao PQ, Huang NY, Zhang WX, et al. Controlling guest conformation for efficient purification of butadiene. Science, 2017, 356: 1193-1196

5 Bereciartua PJ, Cantín Á, Corma A, et al. Control of zeolite framework flexibility and pore topology for separation of ethane and ethylene. Science, 2017, 358: 1068-1071

6 Meng X, Xiao FS. Green routes for synthesis of zeolites. Chem Rev, 2014, 114: 1521-1543

7 Xie B, Song J, Ren L, et al. Organotemplate-free and fast route for synthesizing beta zeolite. Chem Mater, 2008, 20: 4533-4535 